\title{
Production of photonic nanojets by using pupil-masked 3D dielectric cuboid
}

Yue, Liyang; Yan, Bing; Monks, James; Wang, Zengbo; Nguyen, Thanh Tung; Vu, DinhLam; Minin, Oleg; Minin, Igor

\section{Journal of Physics D: Applied Physics}

DOI:

10.1088/1361-6463/aa61cb

Published: 01/01/2017

Peer reviewed version

Cyswllt i'r cyhoeddiad / Link to publication

Dyfyniad o'r fersiwn a gyhoeddwyd / Citation for published version (APA):

Yue, L., Yan, B., Monks, J., Wang, Z., Nguyen, T. T., Vu, D., Minin, O., \& Minin, I. (2017).

Production of photonic nanojets by using pupil-masked 3D dielectric cuboid. Journal of Physics D: Applied Physics, 50, [175102]. https://doi.org/10.1088/1361-6463/aa61cb

\footnotetext{
Hawliau Cyffredinol / General rights

Copyright and moral rights for the publications made accessible in the public portal are retained by the authors and/or other copyright owners and it is a condition of accessing publications that users recognise and abide by the legal requirements associated with these rights.

- Users may download and print one copy of any publication from the public portal for the purpose of private study or research.

- You may not further distribute the material or use it for any profit-making activity or commercial gain

- You may freely distribute the URL identifying the publication in the public portal?
}

Take down policy

If you believe that this document breaches copyright please contact us providing details, and we will remove access to the work immediately and investigate your claim. 


\title{
Production of photonic nanojets by using pupil-masked 3D dielectric cuboid
}

Liyang Yue ${ }^{1}$, Bing Yan ${ }^{1}$, James N Monks ${ }^{1}$, Zengbo Wang ${ }^{1}$, Nguyen Thanh Tung ${ }^{2}$, Vu Dinh Lam ${ }^{2}$, Oleg Minin $^{3}$, and Igor Minin ${ }^{4}$

${ }^{1}$ School of Electronic Engineering, Bangor University, Bangor, LL57 1UT, UK

${ }^{2}$ Institute of Material Science, Vietnam Academy of Sciences and Technology, 18 Hoang Quoc Viet, Cau Giay, Hanoi, Vietnam

${ }^{3}$ National Research Tomsk State University, Lenin Ave., 36, Tomsk, 634050, Russia

${ }^{4}$ National Research Tomsk Polytechnic University, Lenin Ave., 30, Tomsk, 634050, Russia

E-mail:1.yue@bangor.ac.uk

\begin{abstract}
Photonic nanojets can be created via a plane wave irradiation of multi-shaped mesoscale dielectric particles, and a waist of full-width at half-maximum (FWHM) smaller than the diffraction limit can be achieved in this process. In this paper, photonic nanojet produced by a pupil-masked 3D dielectric cuboid lens is numerically investigated under the irradiation of $532 \mathrm{~nm}$ wavelength plane wave. It is found that pupil-masked cuboid lens is not only able to produce photonic nanojets with shorter FWHMs, but also increase its maximal intensity at certain masking ratios on receiving surface. This phenomenon is different from the result of spherical-lens reported in previous publications, and is attributed to convergence of power flow and near-field numerical aperture (NA) increase after analysis of simulated power flow diagrams.
\end{abstract}

\section{Introduction}

Fundamental Rayleigh criterion set strict limitations to observe objects with dimension smaller than the wavelength used to probe them, and the diffraction profiles of the two objects would overlap and cannot be distinguished anymore in this scenario [1-3]. Several research groups around the world are now showing a growing movement in the attempt to overcome the diffraction limit through focusing of radiation in a spot smaller than Airy disk using near-field optics, such as microspheres, which can explore the optical phenomena occurring at subwavelength distance from scattering objects. Regarding optical absorption and scattering by a homogeneous cluster of spherical particles is described by the Lorenz-Mie theory - a particular solution of Maxwell equations, meanwhile treatment of a homogeneous refractive microlens, e.g. a spherical or cylindrical microparticle, is considered as a special case for Lorenz-Mie's solution of Maxwell equations [4,5]. Hence, in 1987, it was theoretically and experimentally verified that a focusing area beyond diffraction limit locates near the shadow surface of the micro-cylinder/sphere, and its peak intensity and radial position depend on the size and refractive index of particle [6]. This phenomenon is later termed as photonic nanojet [7-10]. Subsequently it was found that photonic jets can be generated not only by micro-spheres/cylinders, but also dielectric mesoscale cuboid particles both in transmitting and reflection modes [11-14]. As a relatively easy fabricated microlens, cuboid microlens has a more circular focal spot and a proportional jet length to the side length and lens volume [15].

Meanwhile, it is known that typical factors that can affect the behaviours of a photonic nanojet are refractive index contrast (with respect to surrounding medium) and geometry and size of particle. However, in reality these conditions are difficult to modify due to restrictions on fabrication and processing technologies. Having the ability to control near-field focusing of a particle is still challenging in a flexible and easy-to-implement manner. Annular apodization and phase masks were applied to solve similar problem in far-field $[1,16]$, and transverse resolution can be improved by using different normalised inner circular mask [17]. Annular pupil plane filter was also employed to tune the imaging properties for confocal microscopies [18]. Nonetheless, near-field optics is more complicated due to the 
influence of evanescent wave and thus, no simplified formulations can be used. The idea to modulate of photonic jet parameters by using dielectric particle and pupil mask was described by Minin et al. in 2015 [19]. Independently, Yan et al. numerically proved that pupil mask is capable to reduce spot size of photonic jet produced by near-field spherical lens in 2016 [20], following with $\mathrm{Wu}$ et al. experimentally verifying the improvement of near-field focusing after pupil-masking of microsphere in a short time [21]. Nevertheless, intensity loss of photonic nanojets is an unavoidable issue for the usage of pupil-mask to reduce the FWHM in these previous reports because of smaller amount of light entering into masked lens.

In this paper, we report that intensity loss of mask shaped photonic nanojets can be eliminated in the case of cuboid lens focusing. Photonic nanojets produced by a pupil-masked 3D dielectric fused silica cuboid are numerically simulated under the irradiation of $532 \mathrm{~nm}$ wavelength plane wave. Modelling result shows that pupil mask with specific masking ratio makes photonic nanojets emitted by cuboid lens simultaneously possess higher enhancement of electric intensity and smaller FWHMs compared to same sized non-masked model, which is different from the established theory for sphere-aided photonic nanojets under the identical modelling environment. Photonic nanojets are analysed via multiple measured parameters, e.g. intensity enhancement, FWHM along $x$ and $y$ axes $\left(\mathrm{FWHM}_{\mathrm{x}}\right.$ and $\left.\mathrm{FWHM}_{\mathrm{y}}\right)$, etc., to exhibit the impact of pupil mask, and physics behind simulation is explored through near-field power flow diagrams (Poynting vector distribution) at typical masking ratio where extra intensity enhancement is found.

\section{Results}

Current model is built by using commercial finite integral technique (FIT) software package - CST Microwave Studio. Triangular grids and tetrahedral meshes are fitted with 'open space' boundary condition along $x, y$ and $z$ directions to approach better accuracy for simulation. $532 \mathrm{~nm}$ wavelength $(\lambda)$ plane wave propagates from $+\mathrm{z}$ to $-\mathrm{z}$ direction, and electric and magnetic fields, representing as $e$ and $h$ in Figure 1 (a), are along $y$ and $x$ respectively. Mesh densities are set to $\lambda / 8$ for model and $\lambda / 6$ for background. The whole model is structured as a fused silica cuboid covered by an aluminium pupil mask, while a same sized and non-masked cuboid is also created as a reference. Values of material properties, refractive index, $n$, and extinction coefficient, $k$, are collected from previous publications ( $n$ $=1.46$ for fused silica and $n=0.88, k=6.47$ for aluminium) [22,23]. Side lengths of cuboid, $L$, and pupil mask, $a$, are normalised to $\lambda$. The studied cuboid structure has identical length, width and height, and pupil mask is a square shaped slab with $40 \mathrm{~nm}$ thickness, as shown in Figure 1 (a). A masking ratio (MR) formula for the receiving surface of cuboid is expressed by (1):

$$
\mathrm{MR}=\frac{a^{2}}{L^{2}} \times 100 \%
$$

Figure 1 (b) shows the statistics of photonic nanojets parameters for multi-dimension cuboids (side length from 1 to $4 \lambda$ ) with a $25 \%$ masking face where plane wave enters the lens body. Due to the fact that electric field intensity, $E$, (strength of any point in electric field, unit: $\mathrm{V} / \mathrm{m}^{2}$ ) is proportional to square of the light amplitude, $E^{2}$ intensity is summarised to exhibit local enhancement at focus behind cuboid in first row. It is shown that non-masked reference models have higher enhancement from $L=$ $1 \lambda$ to $1.5 \lambda$, then masked models starts to exhibit advantages on intensity enhancement. At $L=1.75 \lambda$ enhancements for masked and non-masked models are at the same level, reaching to 14.22 and 14.29 respectively. Peak of the curve for masked model is 18.05 at $2 \lambda$, with the difference becoming larger for masked and non-masked models. Two large gaps are shown at models with $L=2.75 \lambda$ and $3 \lambda$. Their enhancements achieve to 15.65 and 15.13 for masked models according to 10.63 and 9.96 for nonmasked model, respectively. After $L=3.25 \lambda$ enhancements of masked models maintain the same level, and non-masked models slowly increase and are slightly higher than that for masked model at same dimension. 
Second and third rows of Figure 1 (b) summarise FWHMs at each focus of multi-dimension cuboids along both transversal $x$ and $y$ axes (marked as FWHM $\mathrm{x}$ and $\mathrm{FWHM}_{\mathrm{y}}$ in Figure 1 (b)). Simplified diffraction limit $=\lambda / 2$, is marked as a benchmark shown as black dashed lines. It is found that all $\mathrm{FWHM}_{\mathrm{x}} \mathrm{S}$ of photonic nanojet for non-masked model (black line) are larger than simplified diffraction limit, however masked model (red line) can provide $\mathrm{FWHM}_{\mathrm{x}} \mathrm{S}$ below this benchmark at the cuboid dimension $L<3 \lambda$. Same trend appears in third row for FWHM $\mathrm{y}$ s, though $\mathrm{FWHM}_{\mathrm{y}} \mathrm{s}$ are normally smaller than $\mathrm{FWHM}_{\mathrm{x}} \mathrm{S}$ at the same cuboid size due to the parallelism between plotted plane - $y z$ and electric field, $E$. Also, a wide peak of non-masked $\mathrm{FWHM}_{\mathrm{y}}$ curve is shown after $L=3 \lambda$, which is related to a dramatic profile change of focus on $y z$ plane from single peak mode to three peaks mode. Pupil mask effectively reduces $\mathrm{FWHM}_{\mathrm{x}}$ and $\mathrm{FWHM}_{\mathrm{y}}$ of photonic nanojet in calculated dimensional range and make many of them smaller than simplified diffraction limit. Smallest waist is obtained by $\mathrm{FWHM}_{\mathrm{y}}$ for masked model at $L=1.25 \lambda, a=0.625 \lambda$ and valued to $0.33 \lambda$, even below diffraction limit $\lambda / 2 n$.

Ellipticities of focus caused by the linear polarization of incoming optical wave are shown in fourth row of Figure 1 (b) to represent the ratio between $\mathrm{FWHM}_{\mathrm{x}} / \mathrm{FWHM}_{\mathrm{y}}$. From its data, it is known that pupil mask is able to spatially optimise ellipticity of photonic nanojet and benefits to deliver a more circular focal spot on $x y$ plane. Ellipticity of a perfect round focus, 1.0, is marked as black dashed line in fourth row. Curve of masked model (red) in fourth row is more approaching to the 1.0 line than that for nonmasked model (black). A relatively circular focus usually has more advantages for the applications on imaging and nanomaterial processing. Meanwhile, focal length of cuboid lens is shortened by pupil mask as well, which is quantified as the distance from focal point to cuboid bottom shown in fifth row in Figure 1 (b). Corresponding shortening is more distinct for cuboids with larger dimensions. For $L=$ $3.5 \lambda$ model difference of focal point to bottom between non-masked and masked cuboids achieves to $2.73 \lambda$, then start narrowing at $L=3.75 \lambda$ and $4 \lambda$.

Briefly, functions of pupil mask coverage applying on cuboid particles are enhancement of focus intensity, increase of focal spot roundness, shortening and control of waist size (FWHMs) and focal length. A typical example - $E^{2}$ field distribution for cuboid dimensioned as $L=2.75 \lambda$ with and without $25 \%$ masking is shown in figure 1 (c). Plane wave direction is from top to bottom. Peak enhancement of intensity for unmasked and masked models are 10.63 and 15.65 respectively in this figure, and photonic nanojets produced by masked cuboid (right) is narrower and closer to cuboid bottom with a higher focus intensity (bright red zone).

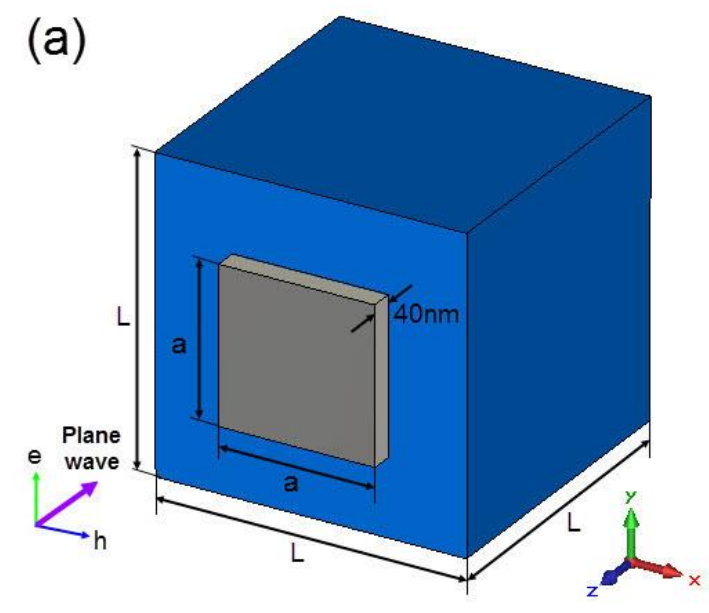



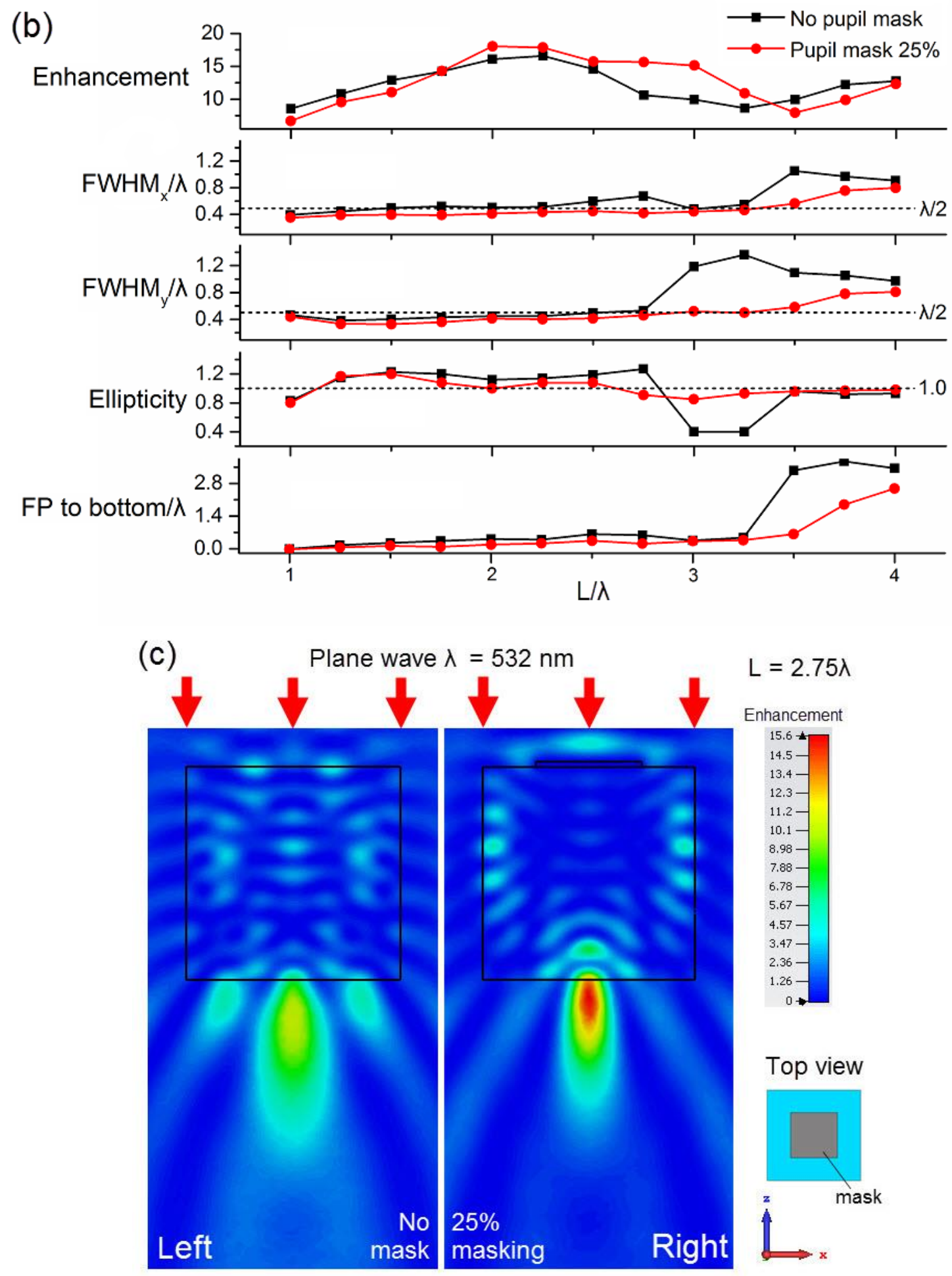

Figure 1 (a) CST model (b) Parameters of photonic nanojets (c) $E^{2}$ field distribution for the cuboid with (Left) and without (Right) 25\% masking

Masking ratio is another factor relating to the parameters of photonic nanojet, e.g. focal point position, FWHM, and peak intensity at focus spot. Figure 2 (a) illustrates $E^{2}$ intensity along $z$ axis at the centre of cuboid $(x=0, y=0)$ for same sized $(\mathrm{L}=2.75 \lambda)$ non-masked reference (black dashed curve) and cuboid with $25 \%, 50 \%$ and $75 \%$ masking. Plane wave propagates in '+' direction from left to right shown in figure 2 (a). Cuboid is placed at 0 position on $z$ axis for modelling, and its space is marked as 
filled pattern in pink from 0 to $1463 \mathrm{~nm}$ on $x$ axis in the figure 2 (a). Basically, all curves form ripples in the cuboid zone, and peak intensity of ripple for non-masked reference is higher than that for masking models in the area close to upper interface of cuboid (left boundary). However, there is a different feature in the area around lower interface (right boundary) that last two ripples of masking models are higher than that for non-masked reference prior the main peak after plane wave exits from cuboid.

Main peaks for multiple masking ratios diverge from their heights and positions. Non-masked reference delivers a main peak at $1782 \mathrm{~nm}$ in the air zone with $10.63 E^{2}$ intensity enhancement, then the curve (black dashed) starts to slowly drop in the modelling space. Curves for other masking models keep the same down trend following a single peak in the air medium, but positions of main peaks are closer to the lower interface. The distance between main peak of $25 \%$ masking and that for non-masked reference is $193 \mathrm{~nm}$ shown in figure 2 (a), and regarding peak is the highest in three masking models arriving to 15.65 intensity enhancement. Once masking ratio is over 25\%, main peak will decline and approach to the lower interface (right boundary) of cuboid, which reflects on the subsequent features 10.49 enhancement with $229 \mathrm{~nm}$ distance and 6.15 enhancement with $316 \mathrm{~nm}$ distance to reference main peak for $50 \%$ and $75 \%$ masking models, respectively. Figure 2 (b) shows the $\mathrm{FWHM}_{\mathrm{x}} \mathrm{s}$ of corresponding peak to masking ratio changes, and a simplified diffraction limit $=\lambda / 2$ is marked in this figure as a width between two solid block line. It is found that all $\mathrm{FWHM}_{\mathrm{x}} \mathrm{S}$ are smaller than the simplified diffraction limit except non-masked reference's. Degradation of peak values is in accordance with the result in figure 2 (a) for enlargement of masking area. FWHM $\mathrm{x}$ are $359 \mathrm{~nm}, 221 \mathrm{~nm}, 223 \mathrm{~nm}$ and $199 \mathrm{~nm}$ for reference, $25 \%$ masking, $50 \%$ masking, and 75\% masking models respectively.

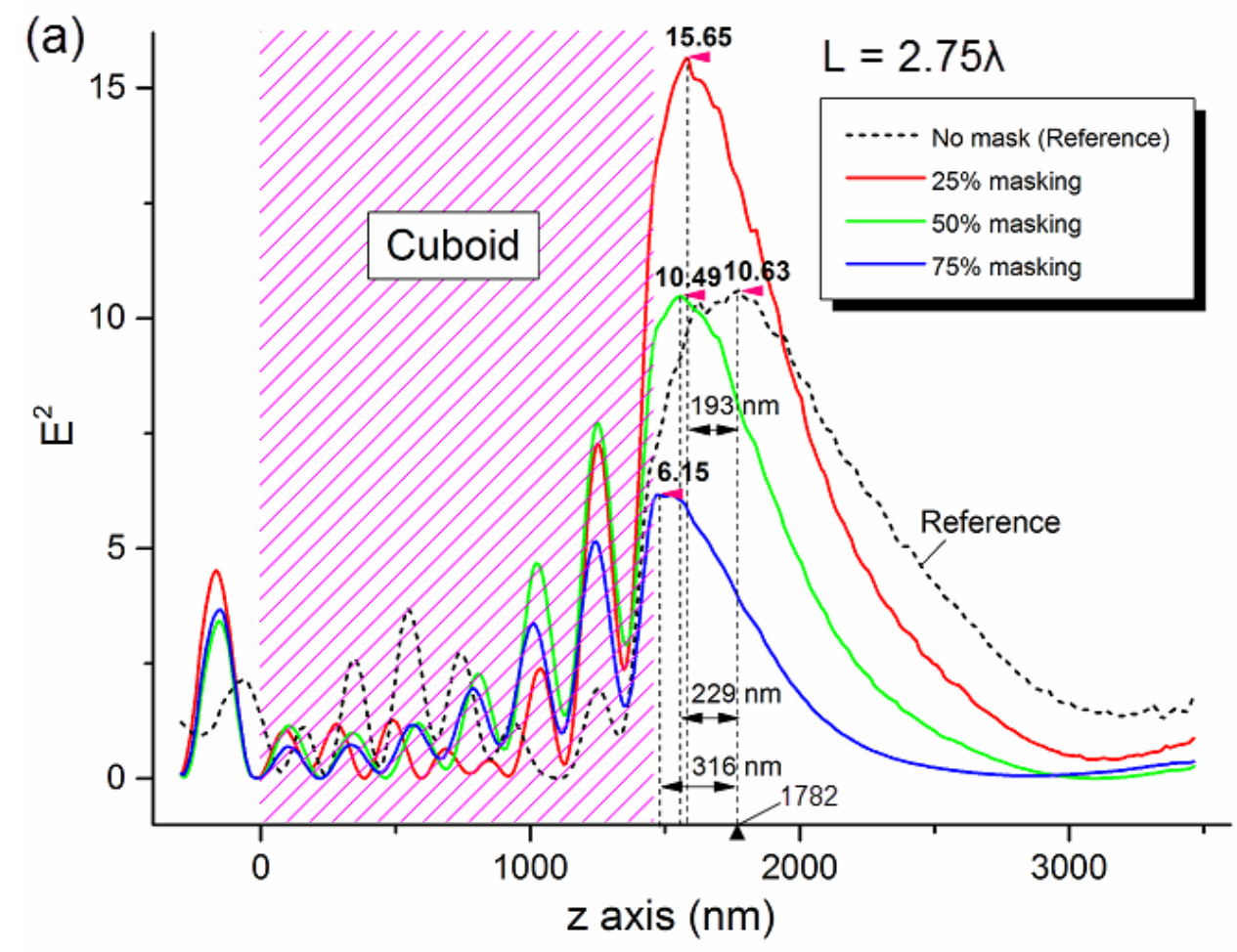




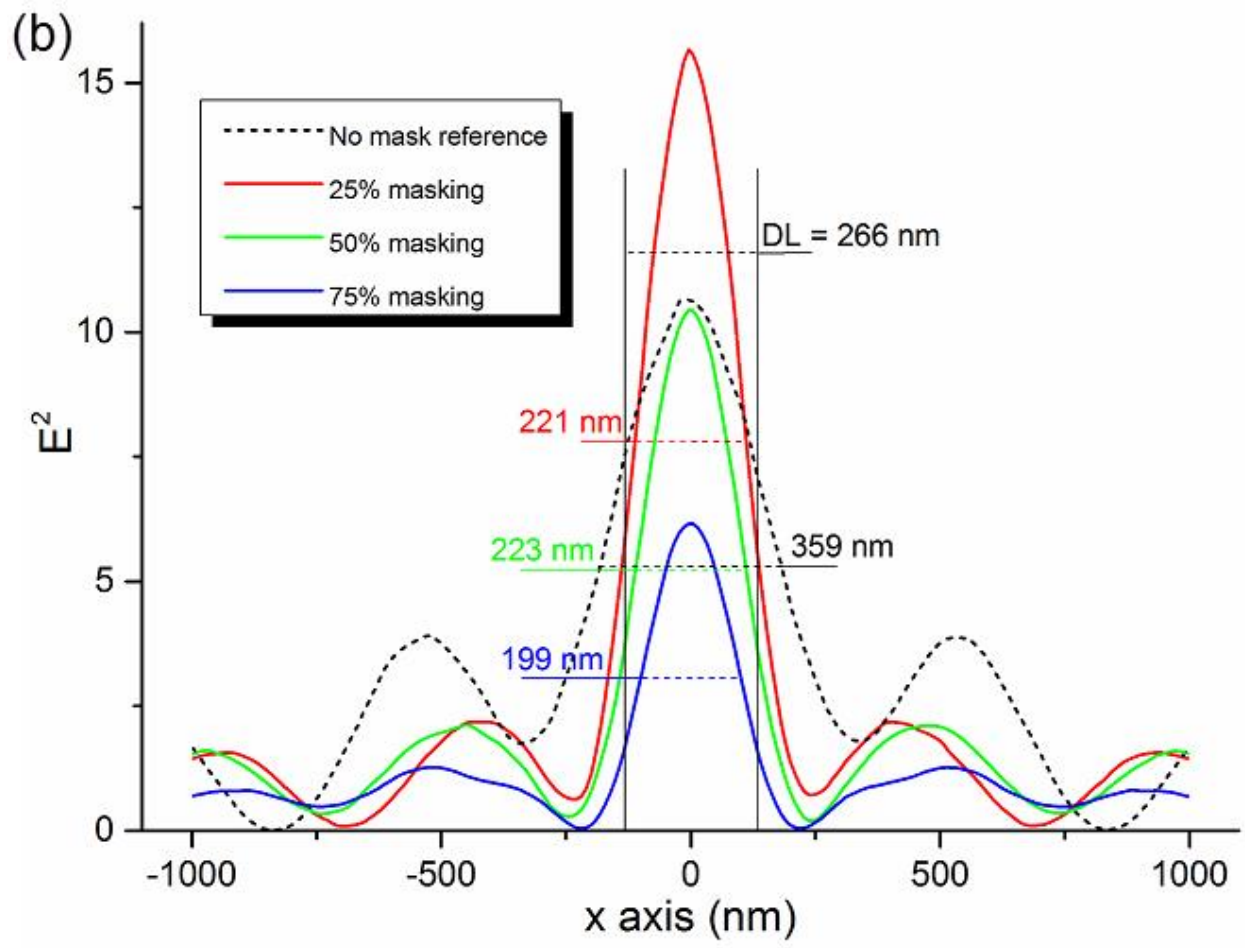

Figure 2 (a) $\mathrm{E}^{2}$ intensities along $\mathrm{z}$ axis at the centre of cuboid $(x=0, y=0)$ for $\mathrm{L}=2.75 \lambda$ masked and non-masked models (b) $\mathrm{E}^{2}$ intensity along $x$ axis and $\mathrm{FWHM}_{\mathrm{x}}$ at focal points position for masked and non-masked models

\section{Discussion}

\subsection{Parameters of photonic nano-jets}

From previous publications [20], it is well known that pupil mask is able to affect parameters of photonic nanojets, moreover, allowing to engineer the focus of near-field lens. Mechanism can be understood as a method to modulate numerical aperture (NA) of near-field optics by using pupil mask. NA of an optical system is a figure to characterise the range of angles which system can accept light, expressed by [1]:

$$
\mathrm{NA}=\mathrm{n} \cdot \sin \theta
$$

where $\theta$ is the maximal half-angle of the cone of light that can enter or exit the lens, meanwhile resolution, $r$, is limited by the NA as well, which is defined as [1]:

$$
\mathrm{r}=\frac{\lambda}{2 \cdot \mathrm{NA}} \approx \frac{\lambda}{2 \cdot \mathrm{n}} \approx \frac{\lambda}{2}
$$

A larger NA enables to deliver a higher resolution based on equation (3). Similar mechanism adapts to applications in near-field optics for development of near-field scanning optical microscope (NSOM) [24]. It is different from the far-field optics that changing of NA for a near-field system is difficult most of time. For a particle lens, 'window' to receive incident light is normally restricted in the central area close to upper boundary and far smaller than the section of particle due to refraction between air and particle material.

Characteristic of a 3D cuboid pairing with a square pupil mask is further intensity enhancement at focal spot of photonic nanojet at certain masking ratio, which is against the established theory working on spherical and circular lens about focusing of incoming light. For apodization effect plain wave is blocked by pupil mask during propagation and energy is consumed in this process, which results in split of power flow stream and large incidence angle of plain wave (high NA) compared to the non-masked model $[20,21]$. A near-field power flow diagram - Figure $3, \mathrm{~L}=3 \lambda$, is helpful to explain above mentioned phenomenon of intensity enhancement. Enhancements of photonic nanojets are 10.63 and 15.65 for non-masked (Figure 3 (a)) and 25\% masking models (Figure 3 (b)), respectively. It is shown 
that a blue lined area is between two angularly arranged high intensity regions (red colour) in the middle of cuboid. Due to the blocking of a pupil mask at the centre of propagation direction, all light enters the particle lens from sides and artificially produce a wider 'window' with larger $\theta$ and NA in Figure 3 (b), which reflects on smaller FWHMs, nearly 1.0 ellipticity, and shorter focal length compared to those for non-masked model, summarising in Figure 1 (b).

\subsection{Intensity enhancement}

Figure 3 (a) shows that three low intensity singular points are at lower part of cuboid (blue colour). Phase trajectories contains 1.5 degrees of freedom in the vicinity of the singular points, and the related clockwise vortex shape represents a stable focus in the phase space [25]. Power flow couples to the other planes through these singular points [26], which results in low field intensity in these areas. Hence, these three singular points properly 'divide' main streams and make three separated high intensity focusing areas below the lower boundary of cuboid.

$25 \%$ masked model provides different power flow streams as shown in Figure 3 (b). Low intensity blue colour takes the central region of cuboid body due to apodization effect caused by pupil mask coverage. In this case, the cuboid may function like a truncated pyramid without loses of photonic jet quality, and rest of power flow rounding the pupil mask make up two straight high intensity streams (red colour) approaching to the left and right boundaries and converging into a 'pocket' in the lower part of cuboid without interference of singular points for non-masked model in Figure 3 (a). For this reason, previous three separated focuses are assembled into one main focus, which cancel out the loss of incoming light and boost intensity enhancement. Larger or smaller masking ratio would result in non-convergence of streams, which makes pupil mask not deliver extra enhancement for photonic nanojets.

Besides, geometry of cuboid decides that it has larger section size compared to a sphere with same volume, and mechanism of its focusing is different from trapping more light in vicinity of lens for spherical particles [26]. Normally there is limited transmission of optical radiation if an object (cuboid) is placed in the path of a propagating wave. However, when the optical transmission exceeds the expected geometrical transmission, enhancement of optical transmission occurs due to the coupled action that transmits more light than that transmitted by the geometrical cross section of the entire cuboid. In addition, if a pupil mask is placed in front of the cuboid, there could be a second enhancement of optical transmission due to the collective action of the optically coupled masks, which is according to the extra enhancement for masked models shown in Figure 1 (b) when $L / \lambda$ is in the range of 2.5-3.25. 

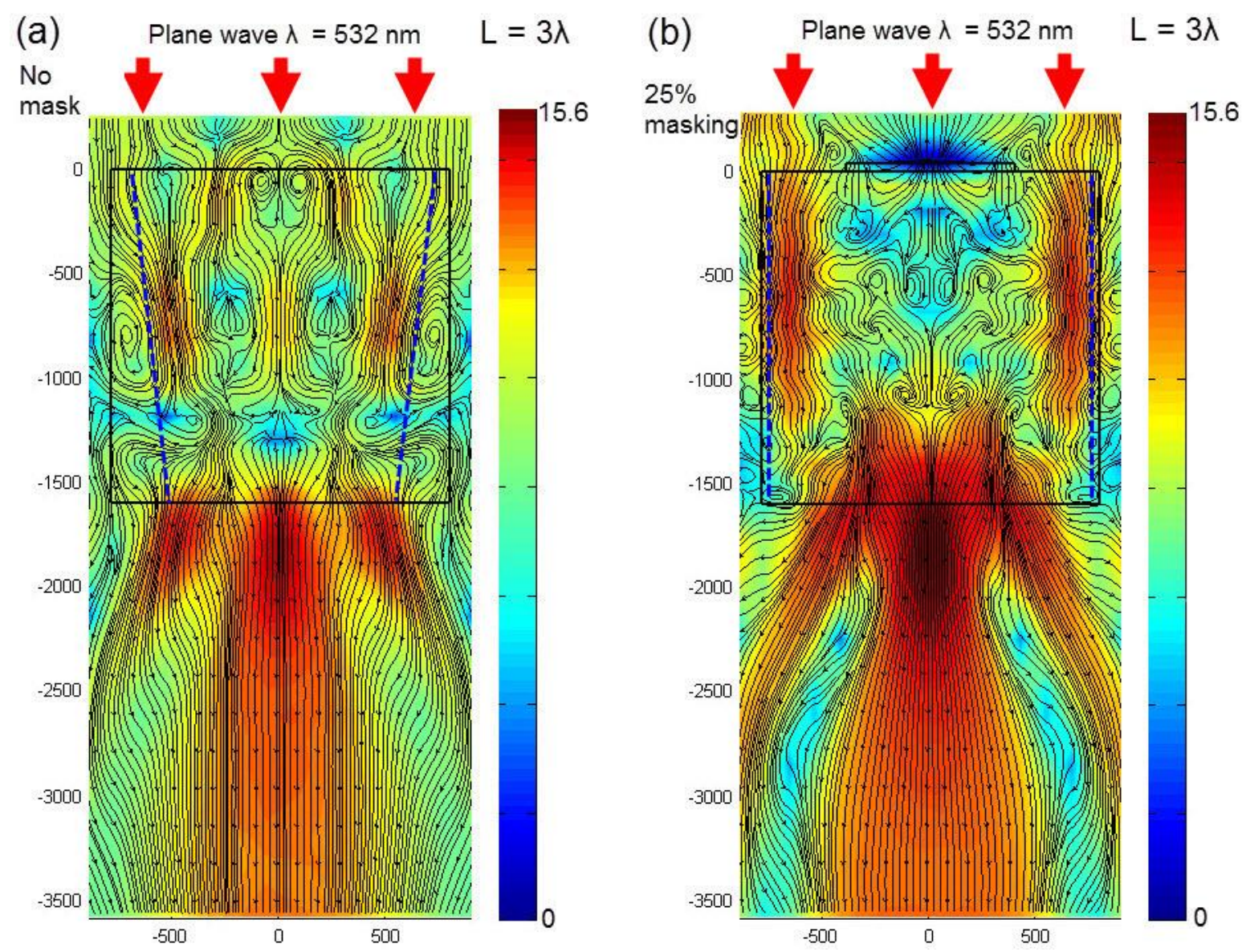

Figure 3 Power flow diagram for cuboid dimensioned with $3 \lambda$ side length without (a) and with (b) a pupil mask on top

\section{Conclusion}

We successfully create a numerical model to simulate production of photonic nanojets using a pupilmasked 3D dielectric cuboid. Regarding pupil mask function on cuboid lens is different from that for spherical lens reported in previous literatures. In this paper, the cuboid-lens-made photonic nanojets after pupil-masking can simultaneously possess higher intensity and smaller FWHM beyond diffraction limit compared to those for non-masked model, which is respectively attributed to convergence of power flow streams and near-field NA increase. Also, focal length and FWHMs of this system can be precisely tuned via adjustment of pupil masking ratio in near-field zone. Therefore, this work is expected to play an important role in the research of super-resolution imaging and material surface processing combing with easy-to-fabricate characteristic of cuboid particle lens.

\section{Acknowledgements}

The authors gratefully acknowledge the financial support provided by the Sêr Cymru National Research Network in Advanced Engineering and Materials (ref: NRNF66 and NRN113), Newton Research Collaboration Programme (ref: NRCP1516/1/153), and the Knowledge Economy Skills Scholarships (KESS 2, ref: BUK289).

\section{References}

[1] Born M and Wolf E 1999 Principles of Optics, 7th ed. (New York: Cambridge University Press)

[2] Jenkins F A and White H E 1951 Fundamentals of Optics, 3rd ed. (New York: McGraw-Hill Primis Custom Publishing)

[3] Allen K W, Farahi N, Li Y, Limberopoulos N I, Walker D E, Urbas A M and Astratov N 2015 Opt. Express 2324484 
[4] Hulst V D 1957 Light Scattering by Small Particles (New York: Courier Dover Publications)

[5] Kreibig U and Vollmer M 1995 Optical properties of metal clusters (Berlin: Springer)

[6] Benincasa D S, Barber P W, Zhang J Z, Hsieh W F, and Chang R K 1987 Appl. Opt. 26 13481356

[7] Chen Z, Taflove A and Backman V 2004 Opt. Express 12 1214-1220

[8] Heifetz A, Kong S C, Sahakian A V, Taflove A, Backman V 2009 J. Comput. Theor. Nanosci. 6 1979-1992

[9] Li X, Chen Z, Taflove A and Backman V 2005 Opt. Express 13 526-533

[10] Ferrand P, Wenger J, Devilez A, Pianta M, Stout B, Bonod N, Popov E and Rigneault H 2008 Opt. Express 16 6930-6940

[11] Minin I V and Minin O V 2014 Vestnik NGU, Ser. Inform. Tekhnol. 459

[12] Pacheco-Pena V, Beruete M, Minin I V and Minin O V 2014 Appl. Phys. Lett. 105084102

[13] Minin I V, Minin O V, Pacheco-Pena V and Beruete M 2015 Opt. Lett. 402329

[14] Minin I V, Minin O V and Geints Y E 2015 Ann. Phys. (Berlin) 527 491-497

[15] Minin I V and Minin O V 2016 Diffractive optics and nanophotonics: resolution below the diffraction limit (Berlin: Springer)

[16] Paeder V, Scharf T, Ruffieux P, Herzig H P, Voelkel R and Weible K 2007 J. EOS-Rapid

Commun. 207005

[17] Sulai Y N and Dubra A 2012 Biomed. Opt. Express. 3 1647-1661

[18] Wilson T and Hewlett S J 1989 J. Mod. Opt. 37 2025-2046

[19] Minin I V and Minin O V 2015 Patent of Russia N 153686

[20] Yan B, Yue L and Wang Z B 2016 Opt. Commun. 370 140-144

[21] Wu M, Chen R, Soh J, Shen Y, Jiao L, Wu J, Chen X, Ji R and Hong M 2016 Sci. Rep. 631637

[22] Malitson I H 1965 J. Opt. Soc. Am. 55 1205-1208

[23] Palik E D 1985 Handbook of Optical Constants of Solids, vol. 1 (Cambridge, Massachusetts:

Academic Press)

[24] Hecht E 2002 Optics (San Francisco: Addison Wesley)

[25] Karlov N V, Kirchenko N A and Luk'yanchuk B S 2000 Laser Thermochemistry: Fundamentals and Applications (Cambridge: Cambridge International Science)

[26] Wang Z B, Luk'yanchuk B S, Hong M H, Lin Y and Chong T C 2004 Phys. Rev. B 70035418 